\title{
Strongly Pseudoconvex Manifolds and Strongly Pseudoconvex Domains
}

\author{
By
}

Shigeo NAKANO* and Takeo OHSAWA*

\section{$\S 1$. Statement of the Result}

A non-compact complex manifold $X$ is called a strongly pseudoconvex (spc) manifold if it is exhausted by a $C^{\infty}$ function which is strictly plurisubharmonic outside a compact set, that is to say, if there exist a proper $C^{\infty}$ map

$$
\dot{\psi}: X \longrightarrow[0, d) \quad(0<d \leqq \infty)
$$

and a constant $c_{0}\left(0<c_{0}<d\right)$ such that the Hermitian matrix $\left(\frac{\partial^{2} \psi}{\partial z^{\alpha} \partial \bar{z}^{\beta}}\right)$ is positive definite at any point $x$ for which $\phi(x)>c_{0}$. Here $(z)$ denotes a holomorphic local coordinate system around $x$. If $X$ and $\phi$ are as above and if we set

$$
X_{u}=\{x \in X \mid \phi(x)<u\} \quad\left(c_{0}<u<d\right),
$$

then $X_{u}$ is relatively compact in $X$ and is itself an spc manifold. We shall call an spc manifold which can be thus represented an $s p c$ domain.

We shall assume that $X$ is connected throughout. The purpose of the present article is to find out a nice sufficient condition for an $\mathrm{spc}$ manifold $X$ to be an spc domain. For the purpose we consider a Hermitian metric

$$
d s^{2}=2 \sum g_{a} \bar{\beta} d z^{n} d \bar{z}^{,}
$$

on $X$, such that

$$
g_{n \bar{\beta}}=\frac{\partial^{2} \psi}{\partial z^{a} \partial \bar{z}^{\beta}} \quad \text { on } \quad X-X_{c_{0}} .
$$

It is clear that there exists such a metric if we replace $c_{0}$ by a bit larger value. It is also clear that the conditions stated below are independent of the choice of such metrics.

(a) $\psi$ is bounded, that is to say $d<\infty$.

(b) With the metric $d s^{2}$, the diameter and the volume of $X$ are finite.

(c) Tensor fields composed of successive derivatives of $\phi$ have bounded magnitudes, the magnitude being counted pointwise with respect to $d s^{2}$ and bound-

Received May 30, 1983. Revised October 20, 1983.

* Research Institute for Mathematical Sciences, Kyoto University, Kyoto 606, Japan. 
edness referring to the change of the point in $X$.

(d) There exists a constant $k_{1}>0$ such that $\sum g_{a} \bar{\beta} u^{\alpha} \bar{u}^{\beta} \geqq k_{1}\left|\sum_{\alpha} \frac{\partial \psi}{\partial z^{\alpha}} u^{\alpha}\right|^{2}$ for $(u)$ $\in C^{n}$ and at every point of $X$. Namely, the length of $\partial \phi$ is bounded.

(e) There exist a positive constant $k_{2}$ and a constant $c_{1}\left(c_{0}<c_{1}<d\right)$ such that

$$
\Lambda \equiv \sum g^{\bar{\beta} a} \frac{\partial \psi}{\partial z^{\alpha}}-\frac{\partial \psi}{\partial \bar{z}^{\beta}} \geqq k_{2} \quad \text { on } \quad X-X_{c_{1}} .
$$

Namely, the length of $\partial \psi$ is larger than a positive constant outside a compact set.

The statement in (c) may not be clear enough, but it will be clear in the course of the proof. If $X$ is an spc domain, conditions (a) $\sim(c)$ are satisfied and condition (d) is realized when we replace $\psi$ by $\exp \phi$. If, moreover, the function $\psi$ is not critical at any point of $\partial X$, then (e) is satisfied.

The main result is :

Theorem. If an spc manifold $X$ with the exhaustion function $\phi$ satisfies the conditions (a) (e) and if $n=\operatorname{dim}_{C} X \geqq 3$, then $X$ is an spc domain, that is to say. there exists an spc manifold $\tilde{X}$ with an exhaustion function $\tilde{\varphi}$ such that sup $\tilde{\varphi}>d$,

$$
\tilde{Y}_{d} \equiv\{x \in \tilde{X}: \tilde{\phi}(x)<d\}
$$

is biholomorphically homeonorphic with $X$ and, after identifying these, we have $\tilde{\varphi} \mid x=$.

The condition $n \geqq 3$ is used in the proof because we make use of a result in $[7]$.

\section{§2. Product Structure as Differentiable Manifolds}

We shall assume that conditions (a) (e) hold for $X$ and $\phi$. Let us take $c$ such that $c_{1}<c<d$ and set $B_{c}=\{x \in X \mid \phi(x)=c\}$, then $B_{c}$ is a compact differentiable manifold of real dimension $2 n-1$ and we have $X-\bar{X}_{c_{1}} \cong B_{c} \times\left(c_{1}, d\right)$ (diffeomorphic). To be more precise, let us consider the family of surfaces $\left\{B_{u} \mid c_{1}<u\right.$ $<d$ \} (defined similarly to $B_{c}$ ) and the vector field orthogonal to these surfaces. The vector field $\sum g^{\bar{\beta} \alpha} \frac{\partial \phi}{\partial \bar{z}^{\beta}} \frac{\partial}{\partial z^{a}}+\sum g^{\bar{\alpha}^{3}} \frac{\partial \phi}{\partial \bar{z}^{\beta}} \frac{\partial}{\partial \bar{z}^{a}}$ is such. We shall normalize this as

$$
\boldsymbol{v}=\frac{1}{2 \Lambda}\left\{\sum g^{\bar{\beta} \alpha} \frac{\partial \psi}{\partial \bar{z}^{\beta}} \frac{\partial}{\partial z^{\alpha}}+\sum g^{\bar{\alpha} \beta} \frac{\partial \psi}{\partial z^{\beta}} \frac{\partial}{\partial \bar{z}^{a}}\right\},
$$

and consider the integral curve $\gamma$ of this vector field. In other words we consider the ordinary differential equation

$$
\frac{d z^{\alpha}}{d u}=\frac{1}{2 \Lambda} \sum g^{\bar{\beta} a} \frac{\partial \psi}{\partial \bar{z}^{\beta}}, \quad \frac{d \bar{z}^{a}}{d u}=\frac{1}{2 \Lambda} \sum g^{\bar{a} \beta} \frac{\partial \psi}{\partial z^{\beta}} .
$$

The effect of the normalization lies in the fact that we have 


$$
\frac{d \psi}{d u}=1
$$

along each integral curve $\gamma$. Thus the parameter $u$ can be interpreted as the value of $\phi$ at the corresponding point of $\gamma$.

We start at the value $c$ of $u$ with the initial condition $\gamma(u)=\xi \in B_{c}$, then the solution will be

$$
z^{a}=z^{n}(u ; \xi)
$$

The solutions for various $\xi$ exist for the interval $c_{1}<u<d$, and by virtue of the general theory of ordinary differential equations, (2.3) gives the diffeomorphism

$$
\begin{gathered}
\Phi: B_{c} \times\left(c_{1}, d\right) \longrightarrow X-\bar{X}_{c_{1}} \\
\Psi \\
(\xi, u) \longmapsto \\
\cup z(u ; \xi),
\end{gathered}
$$

$B_{c} \times u$ being mapped onto $B_{u}$.

We can take the differentiable manifold $B_{c} \times(c, d+\varepsilon)$ and patch it together with $X$ according to the rule $\Phi$ above, then we obtain a differentiable manifold $M$ which contains $X$ as an open submanifold. $X$ is relatively compact in $M$ and the boundary $\partial X$ of $X$ in $M$ is nothing but the subset $B_{c} \times d$ of $B_{c} \times(c, d+\varepsilon)$. $M$ can be considered as a candidate for a manifold which makes $X$ an spc domain. In fact, using conditions (c) and (e) we can prove the following proposition. We omit its proof because it will be routine to the readers. This can also be seen from Proposition $3^{\prime}$ and what follows.

Proposition 0. The completion $\hat{X^{\prime}}$ of $X$ with respect to the distance induced by $d s^{2}$ is nothing but the closure $\bar{X}=X \cup \partial X$ of $X$ in $M$.

\section{$\S 3$. Analysis on Metric Tensors}

On $B_{c}$ we take a coordinate neighbourhood $U$ and differentiable local coordinates $\left(\xi^{1}, \cdots, \xi^{2 n-1}\right)$ on $U$, then

$$
\left(\xi^{1}, \cdots, \xi^{n-1}, \xi^{2 n}\right) \quad \text { where } \xi^{2 n}=u
$$

is a coordinate system on $U \times(c, d+\varepsilon)$ and, if we restrict to the domain $c<\xi^{2 n}$ $<d$, it is a differentiable coordinate system on $\Phi(U \times(c, d)) \subset X$. We shall examine the Riemannian metric $d s^{2}$ a bit closely, in terms of this coordinate system. Set

$$
d s^{2}=\sum_{\imath, \jmath=1}^{2 n} G_{\imath \jmath}(\xi) d \hat{\xi}^{i} d \xi^{\jmath}, \quad G_{\imath \jmath}=G_{\jmath i},
$$

then $G_{i j}(\xi)$ is nothing but the inner product $\left(\partial / \partial \xi^{i}, \partial / \partial \xi^{j}\right)$ of the vector fields $\partial / \partial \xi^{i}$ and $\partial / \partial \xi^{j}$ at the point $(\xi)$. We first consider a vector field $\boldsymbol{v}=\sum a^{i} \partial / \partial \hat{\xi}^{i}$ ( $a^{i}$ being constants) and consider the inner product $(\boldsymbol{v}, \boldsymbol{v})$ as a function of $u=$ 
$\xi^{2 n}$, other coordinates being considered as auxiliary parameters. Set

$$
\varphi(u)=(\boldsymbol{v}, \boldsymbol{v})=2 \sum g_{a} \bar{\beta}(z, \bar{z}) \boldsymbol{v}\left(z^{\alpha}\right) \boldsymbol{v}\left(\bar{z}^{3}\right)
$$

and calculate $\frac{d \varphi}{d u}$ according to the differential equation (2.1). We have, from (2.1),

$$
\begin{aligned}
\frac{d}{d u} \boldsymbol{v}\left(z^{a}\right) & =\boldsymbol{v}\left(\frac{1}{2 \Lambda} \sum g^{\bar{\beta} \alpha} \frac{\partial \psi}{\partial \bar{z}^{\beta}}\right) \\
& =\Sigma\left(\boldsymbol{v}\left(z^{\alpha}\right) \frac{\partial}{\partial z^{\alpha}}+\boldsymbol{v}\left(\bar{z}^{\alpha}\right) \frac{\partial}{\partial \bar{z}^{\alpha}}\right)\left(\frac{1}{2 \Lambda} \sum g^{\bar{\beta} \alpha} \frac{\partial \psi}{\partial \bar{z}^{\beta}}\right),
\end{aligned}
$$

and its conjugate, and this implies that the components of $\nabla_{u} v$ are linear combinations of those of $v$, with tensor field composed of derivatives of $\phi$ as coefficients. Here $\boldsymbol{u}$ denotes the vector field $\partial / \partial \xi^{2 n}$ on $U \times(c, d)$ and $\nabla_{\boldsymbol{u}}$ denotes the covariant differentiation along $\boldsymbol{u}$. Then

$$
\frac{d \varphi(u)}{d u}=\nabla_{\boldsymbol{u}}(\boldsymbol{v}, \boldsymbol{v})=2\left(\nabla_{\boldsymbol{u}} \boldsymbol{v}, \boldsymbol{v}\right)
$$

and we have the following inequality

$$
-L_{1} \cdot \varphi(u) \leqq \frac{d \varphi(u)}{d u} \leqq L_{1} \cdot \varphi(u),
$$

with a certain constant $L_{1}>0$. This implies that

$$
\begin{array}{r}
\varphi(c) \exp \left(-L_{1}(u-c)\right) \leqq \varphi(u) \leqq \varphi(c) \exp \left(L_{1}(u-c)\right) \\
\text { for } c<u<d .
\end{array}
$$

We can take $U$ and $(\xi)$ so that $\left(\xi^{1}, \cdots, \xi^{2 n-1}\right)$ are local coordinates on a neighbourhood of $\bar{U}$. This being done, $\varphi(c)$ is bounded on $U \times c$, and is uniformly bounded in $v$ if we put the condition $\sum_{i}\left(a^{i}\right)^{2} \leqq 1$. This means that we have constants $L_{2}$ and $L_{3}$ such that

$$
0<L_{2} \leqq \sum_{i, j=1}^{2 n} G_{i j}(\xi) a^{i} a^{j} \leqq L_{3} \quad \text { on } \quad U \times(c, d),
$$

$L_{2}$ and $L_{3}$ being independent of $\left(a^{i}\right)$ provided $\Sigma\left(a^{i}\right)^{2} \leqq 1$.

From this we draw the following conclusion immediately.

Proposition 1. The entries of the metric tensor $G=\left(G_{i j}\right)$ with respect to the coordinate system $(\xi)$ are bounded functions on $U \times(c, d)$. $\operatorname{det} G$ remains away from 0 there and the entries of $G^{-1}$ are also bounded. We have $L_{4} d v \leqq d \xi^{1} \cdots d \xi^{2 n} \leqq L_{5} d v$ with $L_{4}, L_{5}>0$.

Next we shall show

Proposition 2. Successive derivatives of $G_{i j}$ are bounded functions on $U \times$ $(c, d)$, bounds depending on the type of derivatives. 
To see this, first take another vector field $w=\sum b^{2} \partial / \partial \xi^{i}$ and examine the boundedness of $\boldsymbol{w}(\boldsymbol{v}, \boldsymbol{v})$. Since

$$
|\boldsymbol{w}(\boldsymbol{v}, \boldsymbol{v})|=2\left|\left(\nabla_{u} \boldsymbol{v}, \boldsymbol{v}\right)\right| \leqq 2\left(\nabla_{w} \boldsymbol{v}, \nabla_{w} \boldsymbol{v}\right)^{1 / 2} \cdot(\boldsymbol{v}, \boldsymbol{v})^{1 / 2},
$$

and since $(\boldsymbol{v}, \boldsymbol{v})$ is bounded, we have only to show the boundedness of $\varphi_{1}=$ $\left(\nabla_{w} v, \nabla_{w} v\right)$. Now

$$
\begin{aligned}
\frac{d \varphi_{1}}{d u} & =\frac{d}{d u}\left(\Gamma_{w} v, \Gamma_{w} v\right)=2\left(\nabla_{u} \nabla_{w} v, \Gamma_{w} v\right) \\
& =2\left(\nabla_{w} \nabla_{u} v, \nabla_{w} v\right)+2\left(\left(\nabla_{u} \nabla_{w}-\nabla_{w} \nabla_{u}\right) v, \nabla_{w} v\right) .
\end{aligned}
$$

As mentioned after the formula (3.4), $\nabla_{\boldsymbol{u}} \boldsymbol{v}$ is the linear combination of $\boldsymbol{v}$ with tensors composed of derivatives of $\phi$ as coefficients. $\nabla_{u} \nabla_{w}-\nabla_{w} \nabla_{u}$ is nothing but the curvature operator. Hence we have the inequality of the form

$$
-L_{6} \varphi_{1}-L_{7} \leqq \frac{d \varphi_{1}}{d u} \leqq L_{6} \cdot \varphi_{1}+L_{7} \quad\left(L_{6}, L_{7}: \text { const }\right) .
$$

Thus we see the boundedness of $\varphi_{1}$, uniformly in $(b)$ provided $\Sigma\left(b^{2}\right)^{2} \leqq 1$.

If we have $r$ vector fields $w_{1}, \cdots, w_{r}$ instead of single $\boldsymbol{w}$, we can prove, by induction on $r$, that $\boldsymbol{w}_{1} \circ \cdots \circ \boldsymbol{w}_{r}(\boldsymbol{v}, \boldsymbol{v})$ is bounded.

This proves that successive derivatives of $G_{i}$ are bounded.

\section{§4. Almost Complex Structures and $C R$ Structures}

Let us consider the tensor $J$ of the almost complex structure induced by the complex structure on $X$. With respect to the holomorphic local coordinate $(z)$, $J$ has the component

$$
\left[\begin{array}{cc}
\sqrt{-1} I_{n} & 0 \\
0 & -\sqrt{-1} I_{n}
\end{array}\right] \quad\left(I_{n}=\text { unit matrix of size } n\right) .
$$

Hence, $|J|^{2}=4 \sum g_{\alpha \bar{\beta}} g^{i \delta} J^{a}{ }_{\delta} \overline{J_{\gamma}}$ and the squares of the magnitude of successive covariant derivatives of $J$ are summable on $X$, by virtue of the conditions (c) and (b). Hence, a fortiori, they are summable on the domain $\Phi(U \times(c, d))$. On $U \times(c, d)$, we consider everything in terms of coordinates $(\xi)$. Then because of Proposition 1, we see the following:

$$
\begin{aligned}
& \int_{U \wedge(c, d)} \sum_{i, j}\left|f^{i}{ }_{\jmath}\right|^{2} d \xi^{1} \cdots d \xi^{2 n}<\infty, \\
& \int_{U<(c, d)} \sum_{i, j, k}\left|\frac{\partial f^{i}{ }_{j}}{\partial \xi^{k}}+\sum_{m} \Gamma_{m k}^{i} f^{m},-\sum_{m} \Gamma_{\jmath k}^{m} \int_{m}^{i}\right|^{2} d \xi^{1} \cdots d \xi^{2 n}<\infty,
\end{aligned}
$$

where $\left\{\int_{j}^{i}\right\}$ stand for the components of $J$ with respect to $(\xi)$, and $\Gamma_{m k}^{\imath}$ are 
those of Christoffel symbols. $G^{j k}, \frac{\partial G_{j k}}{\partial \xi^{l}}, \cdots$ are all bounded on $U \times(c, d)$, therefore we see that $f^{i}{ }_{\jmath}, \frac{\partial f^{i}{ }_{j}}{\partial \xi^{k}}, \frac{\partial^{2} f_{j}^{i}{ }_{j}}{\partial \xi^{k} \partial \xi^{l}}, \cdots$ are all square summable on $U \times(c, d)$. Now let us make use of the following Lemmas, Lemma 1 being the well known Sobolev imbedding theorem. (See for example [2], p. 30 Th. 11.1 and the problem which follows.) Lemma 2 is elementary.

Lemma 1. Consider a bounded domain $\Omega \times(c, d)$ in $\boldsymbol{R}^{m}$, where the boundary of $\Omega$ is a $C^{\infty}$-smooth manifold of dimension $m-2$. If $f$ is a function in the Sobolev space of order $k$ in $\Omega \times(c, d)$, then $f$ is of class $C^{l}$ in $\Omega \times(c, d)$ for $l \leqq k-\left[\frac{m}{2}\right]-1$. Moreover $f$ can be extended as a $C^{l}$-function beyond each regular boundary point of $\Omega \times(c, d)$.

Lemma 2. Notations being as above, if a function $f$ on $\Omega \times(c, d)$ can be extended for any $l>0$, to a function of class $C^{l}$ on $\Omega \times\left(c, d+\varepsilon_{l}\right), \varepsilon_{l}$ being a positive number, then $f$ can be extended to $\Omega \times(c, d+\varepsilon)$ as a function of class $C^{\infty}$, for some $\varepsilon>0$.

By virtue of these Lemmas we can assert the following

Proposition 3. If we replace $U$ by a bit smaller neighbourhood, the components $f^{i}{ }_{j}$ of the almost complex structure tensor can be extended to functions of class $C^{\infty}$ on $U \times(c, d+\varepsilon)$, for some $\varepsilon>0$.

Proposition $3^{\prime}$. The components $G_{i j}$ of the metric tensor can be extended to functions of class $C^{\infty}$ on $U \times(c, d+\varepsilon)$.

We can cover $B_{c}$ by coordinate neighbourhoods $U_{\nu}$ for which Proposition 3 holds. Thus $\left\{U_{\nu} \times(c, d+\varepsilon)\right\}$ may be considered as an open covering of $M$. Making use of a partition of unity, we can extend the tensor field $J$ on $X$ to that on $M$, of class $C^{\infty}$, which we still denote by $J$. We don't know if $J^{2}=-i d$ holds on $M-\bar{X}$, but this relation holds at points of $\bar{X}$. The metric can also be extended to $M$ as a Riemannian metric of class $C^{\infty}$.

For $u$ with $c<u<d, B_{u}=\{x \in X \mid \phi(x)=u\}$ has the $C R$ structure induced by the complex structure of $X$. To give the $C R$ structure is to give the $C$-subbundle $T_{u}^{\prime \prime}$ of the complexified tangent bundle $C T B_{u}$ as

$$
T_{u}^{\prime \prime}=\left.T_{X}^{\prime \prime}\right|_{B_{u}} \cap C T B_{u},
$$

where $T_{X}^{\prime \prime}$ denotes the bundle of tangent vectors of type $(0,1)$. It can also be described in terms of the tensor $J$. Since $J$ is extended to $\partial X$ (taken in $M$ ), we have a $C R$ structure on $\partial X$ too, the limit structure of those on $B_{u}$ 's.

Proposition 4. The limit $C R$ structure on $\partial X$ is strongly pseudoconvex.

Proof. Let us take $u(<d)$ and consider the $C R$ strucure on $B_{u}$. Take a 
point of $B_{u}$ and holomorphic local coordinates $(z)$ in a neighbourhood. Without loss of generality, we can assume that $\partial \psi / \partial z^{n} \neq 0$ in this neighbourhood. Then $\left(T_{u}^{\prime \prime}\right)_{x}$ is generated by

$$
u_{\beta}=\left(\frac{\partial \psi}{\partial \bar{z}^{n}}\right) \partial / \partial \bar{z}^{\beta}-\left(\frac{\partial \psi}{\partial \bar{z}^{\beta}}\right) \partial / \partial \bar{z}^{n} \quad(\beta=1, \cdots, n-1)
$$

and

$$
w_{0}=\sqrt{-1}\left\{\left(\frac{\partial \psi}{\partial z^{n}}\right)^{-1} \frac{\partial}{\partial z^{n}}-\left(\frac{\partial \psi}{\partial \bar{z}^{n}}\right)^{-1} \frac{\partial}{\partial \bar{z}^{n}}\right\}
$$

can be taken as a base of the real subbundle $F$ of $C T B_{u}$, for which $C T B_{u}=$ $T_{u}^{\prime \prime} \oplus \bar{T}_{u}^{\prime \prime} \oplus \boldsymbol{C} F$ holds. By direct computation we have

$$
\left[u_{a}^{\prime}, \bar{w}_{3}\right] \equiv \sqrt{ }-1 c_{3,} w_{0} \bmod \left(w_{1}^{\prime}, \cdots, w_{n-1}, \bar{w}_{1}, \cdots, \bar{u}_{n-1}{ }^{\prime} .\right.
$$

where

$$
\begin{aligned}
c_{, 3 \bar{a}}= & \frac{\partial^{2} \psi}{\partial z^{\beta} \partial \bar{z}^{\alpha}}-\frac{\partial^{2} \psi}{\partial \bar{z}^{a} \partial z^{n}} \frac{\partial \psi}{\partial z^{\beta}}\left(\frac{\partial \psi}{\partial z^{n}}\right)^{-1}-\frac{\partial^{2} \psi}{\partial \overline{\bar{z}}^{\beta} \partial z^{n}}-\frac{\partial \dot{\psi}}{\partial \bar{z}^{a}}\left(\begin{array}{c}
\partial \varphi^{i} \\
\hat{\partial} \bar{z}^{\prime-}
\end{array}\right)^{-1} \\
& +-\frac{\partial^{2} \psi}{\partial z^{n} \partial \bar{z}^{n}}\left(\frac{\partial \psi}{\partial z^{n}} \frac{\partial \psi}{\partial \bar{z}^{n}}\right)^{-1} \frac{\partial \psi}{\partial z^{\beta}} \frac{\partial \psi}{\partial \bar{z}^{a}} .
\end{aligned}
$$

To say that the $C R$ structure on $B_{u}$ is strongly pseudoconvex is nothing but to say the Hermitian form $\sum_{\beta, a=1}^{n-1} c_{, 3 \bar{\alpha}} \xi^{3} \bar{\xi}^{a}$ is positive definite. But it can be seen that this Hermitian form is nothing but the restriction of the Hermitian form $d s^{2}$ onto the subspace $\left(\bar{T}_{u}^{\prime \prime}\right)_{x}$. Hence it is positive definite and, as we have seen in Proposition $3^{\prime}$, the inner product $d s^{2}$ (or the matrix $\left(G_{\imath \jmath}\right)$ ) remains positive definite on $\partial X$. Thus we see that the limit structure is again strongly pseudoconvex.

\section{$\S 5$. Limit $C R$ Structure and Original Complex Structure}

We shall show that the $C R$ holomorphic functions on $\partial X$ are nothing but the boundary values of holomorphic functions on $X-\bar{X}_{u}$ (for some $u<d$ ). Namely we shall prove the following proposition:

Proposition 5. Suppose $\operatorname{dim} X=n \geqq 2$ in addition to the prevous conditions. Given a $C^{\infty}$-function $f$ on $\partial X$ which is $C R$ holomorphic, we can find an open neighbourhood $V$ of $\partial X$ on $M$ and $a C^{\infty}$-function $\tilde{f}$ on $\bar{X} \cap V$ such that $\tilde{f}$ is holomorphic in $X \cap V$ and $\tilde{f} \tilde{i}_{0 . \mathrm{Y}}=f$.

For the proof we shall first establish a Lemma. If we restate the situation: $M$ is a $2 n$-dimensioned manifold with a Riemannian metric $d s^{2}$ of class $C^{\infty}(n \geqq 2)$ and $\psi: M \rightarrow[0, d+\varepsilon)$ is a proper $C^{\infty}$-map. $X=\{x \in M \mid \psi(x)<d\}$ and $d \psi(x) \neq 0$ for $\phi(x)=d$. $X$ has a complex structure for which $\phi$ is strictly plurisubharmonic outside $Y_{c_{1}}-\left\{x \in X \mid \psi(x)<c_{1}\right\}$ and for which $d s^{2}$ is Hermitian. 
We shall further assume that $X$ has a Kähler metric $d \sigma^{2}=2 \sum \Gamma_{a} \bar{\beta} d z^{a} d \bar{z}^{3}$, for which

$$
\left(\frac{\hat{\sigma}^{2} \lambda(\phi)}{\hat{\partial} z^{a} \partial \bar{z}^{\beta}}\right) \leqq\left(\Gamma_{a \bar{\beta}}\right) \leqq B\left(\frac{\partial^{2} \lambda(\phi)}{\partial z^{\alpha} \partial \bar{z}^{\beta}}\right) \quad \text { on } \quad X-X_{c_{2}},
$$

where $c_{2}$ is a constant $c_{1}<c_{2}<d, B$ is a positive constant and $\lambda$ is a non-decreasing convex function of class $C^{\infty}$ on $(-\infty, d)$, with $\lambda \equiv 0$ on $\left(-\infty, c_{1}\right)$ and $=$ $(d-\tau)^{-1}$ for $c_{2}<z<d$.

Let us consider the space $C_{0}^{0, q}(X)$ of the $C^{\infty}$ differential forms of type $(0, q)$, with compact supports in $X$. We introduce two kinds of inner product $(\varphi, \xi)$ and $(\varphi, \xi)_{\lambda}$ by

$$
\begin{aligned}
(\varphi, \xi) & =\int_{X} \varphi_{\wedge} \overline{* \xi} \\
(\varphi, \xi)_{\lambda} & =\int_{X} \lambda(\phi) \varphi_{\wedge} \overline{\dot{\xi} \psi},
\end{aligned}
$$

where $*$ and $\therefore$ denote the formation of the adjoint forms with respect to $d s^{2}$ and $d \sigma^{2}$ respectively. We denote the completions of $C_{0}^{0, q}(X)$ with respect to $($, and $(,)_{\lambda}$ by $L^{q}(X)$ and $L^{q}(X, \lambda)$ respectively. We have $L^{q}(X, \lambda) \subset L^{q}(X)$ as sets of measurable forms. We also introduce into $C_{0}^{q}(M)$, the space of $q$-forms on $M$ with compact supports in $M$, the inner product (,) as in the first formula of (5.2) $X$ being replaced by $M$, and denote the completion by $L^{q}(M)$.

An element $r_{1}$ of $L^{q}(X)$ can be extended to a form $\tilde{\eta}$ on $M$ by setting $\tilde{\gamma}=0$ at points of $M-X$, and gives an element of $L^{q}(M)$. Thus $L^{q}(X) \subset L^{q}(M)$. It should also be noted that $d \sigma^{2}$ is complete because of (5.1). (Proof is the same as [4], Prop. 1.)

Lemma 3. Lnder these circumstances, if $\varphi$ is an element of $L^{q}(X, \lambda)$ with $\overline{\hat{o}} \varphi=0$ in the sense of distributions and if $0<q<n$, then there exists an element $\eta \in L^{q-1}(X, \lambda)$ such that $\varphi=\bar{\partial} \eta$.

Proof. In the spaces $L^{r}(X, \lambda), r=0,1,2, \cdots$ we consider the operator $\overline{\hat{o}}$ and its adjoint $\bar{\partial}_{\dot{\lambda}}^{*}$. Then we have, in general,

$$
L^{q}(X, \lambda)=H \oplus\left[R_{\bar{\partial}}\right] \oplus\left[R_{\bar{\partial}_{\lambda}}\right],
$$

where $R_{\bar{\partial}}, R_{\bar{\partial}_{\bar{\lambda}}}$ denote the ranges of $\bar{\partial}, \bar{\partial}_{\lambda}^{*}$ respectively, [ ] denotes the closure in the Hilbert space and $H=\left\{\varphi \in L^{q}(X, \lambda) \mid \vec{\partial} \varphi=0, \bar{o}_{\lambda}^{*} \varphi=0\right\}$. If we can show that $H=\{0\}$ and $\left[R_{\bar{o}}\right]=R_{\bar{\partial}}$, then we are through.

We define the Laplace-Beltrami operator $\square_{\lambda}$ by $\square_{\lambda}=\overline{\hat{o}} \circ \bar{\partial}_{\lambda}^{*}+\bar{\partial}_{\lambda}^{*} \circ \overline{\hat{o}}$. Then as in [5], formula (56) we have, for $\varphi \in C_{0}^{0, q}(X)$,

$$
\square_{\lambda} \varphi=-\Lambda e(\chi)_{\varphi}+\hbar^{-1} \square_{\lambda} \operatorname{si} \varphi,
$$

where $\chi=-\sqrt{ }-\overline{1} \partial \bar{\partial} \lambda(\psi)$ and $\Lambda$ denotes the adjoint of the exterior multiplication by the Kähler form. From this we obtain 


$$
(\bar{\delta} \varphi, \bar{\partial} \varphi)_{\lambda}+\left(\bar{\delta}_{\lambda}^{*} \varphi, \bar{\delta}_{\lambda}^{*} \varphi\right)_{i} \geqq-\left(\Lambda e(\chi)_{\varphi}, \varphi\right)_{\lambda},
$$

and, if we take (5.1) into account, we can derive, as in [5], 62! the inequality

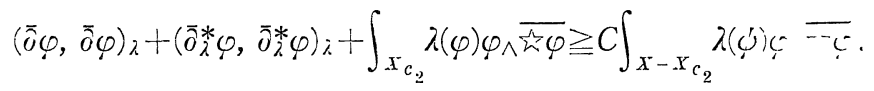

for $\varrho \equiv C_{0}^{0, q}\left(X^{\top}\right)$, where $C$ is another constant.

For an element $\xi$ of $L^{q}(X, \lambda)$ which belongs to the intersection of the domains of $\bar{\partial}$ and $\bar{\partial}_{\lambda}^{*}$, the formula (5.5) still holds good. Hence if $\xi \in H$, then $-(\Lambda e(\chi) \xi, \xi)_{\lambda}$ $=0$. But $\chi$ is negative semi-definite and strictly negative on a non-empty open set. Hence we have $\xi=0$ on this set. (The integrand of $\left(\Lambda e^{\prime} \%\right) \xi \xi_{i}, \lambda$ is negative semi-definite and strictly negative when $\chi$ is so. This can be seen most easily when we take an orthonormal base for differentials of type (0.1), in which coefficients of $\chi$ is diagonal.) By a theorem of Aronszajn on the unique extension of harmonic forms, we have $\xi=0$ on the whole $X$. Thus we have $H=\{0\}$.

Next, proposition 1.2 of [6] shows that (5.6) implies $\left[R_{\bar{\sigma}}\right]=R_{\bar{g}}$.

Proof of Proposition 5. First we shall prove our proposition in case the condition of Lemma 3 is satisfied.

Take a $C R$ holomorphic function $f$ on $\partial X$. As the proof of Theorem 2.3.2' in [3] shows, we can extend $f$ to a $C^{\infty}$-function $\tilde{f}_{1}$ in a neighbourhood $V$ of $\hat{o} X$ in $M$, so that $\bar{\partial} \tilde{f}_{1}=(d-\psi)^{2} \cdot g$, where $g$ is a l-form on $V$ of class $C^{\infty}$. We extend $\tilde{f}_{1}$ to the whole $X$. Then $\bar{\partial} \tilde{f}_{1}$ belongs to $L^{1}(X, \lambda)$. We can apply Lemma 3 and can solve

$$
\bar{\partial} v=\bar{\partial} \tilde{f}_{1}
$$

where $v \in L^{0}(X, \lambda)$.

If we can show that the boundary value of $v$ on $\partial X$ is equal to zero, then $\tilde{f}=\tilde{f}_{:}-v$ satisfies the requirement of the Proposition. ( $v$ is extended to $M$ by setting $r=0$ outside $X$.) To see this let us denote the extended function by $\tilde{\imath}$. Then $\tilde{\imath} \in L^{0}(M)$. We extend the operator $\bar{\partial}$ on $X$ to a differential operator $D$ on $M$. This can be done because the almost complex structure tensor is extended. Take a monotonous $C^{\infty}$-function $\rho$ of $t \in \boldsymbol{R}$, which is $=1$ for $t<-1,=0$ for $t \geqq 0$ and set $\rho_{n}(x)=\rho(n(\phi(x)-d))$. Now for an element $\left.r \equiv C_{1} ; M\right)$, we have

$$
\begin{aligned}
(D \tilde{\imath}, \eta)_{X I} & =\left(\tilde{v}, D^{*} \eta\right)_{X}=\left(v, D^{*} \eta\right)_{X} \\
& =\lim _{n \rightarrow \infty}\left(\rho_{n} \imath^{\prime}, D^{*} \eta\right)_{X}=\lim _{n \rightarrow \infty}\left(\overline{\hat{v}}\left(\rho_{n} v\right), \eta\right)_{X} .
\end{aligned}
$$

Here $\overline{\bar{o}}\left(\rho_{n} v^{2}\right)=\rho_{n} \overline{\bar{\partial}} \tilde{f}_{1}+\bar{\partial} \rho_{n} \cdot \tilde{v}$ and the support of $\bar{\partial} \rho_{n}$ is contained in $\mathrm{I}-X_{d-1 / n}$ and $\left|\bar{\partial} \rho_{n}\right| \leqq n$ (const.), while $v$ is in $L^{0}(X, \lambda)$. Hence we conclude that $\bar{\partial}\left(\rho_{n} v\right)$ converges to $\bar{\partial} \tilde{f}_{1}$ in $L^{1}(X)$. Thus we have proved that $D \tilde{v}$ is locally square summable and that 


$$
D \tilde{v}=\left\{\begin{array}{lll}
\overline{\hat{o}} \tilde{f} & \text { on } & X \\
0 & \text { on } & M-X .
\end{array}\right.
$$

Because of the condition on $\bar{\partial} \tilde{f}_{1}$, the right hand side is a form of class $C^{1}$ on $M$, while the symbol of $D$ remains non-degenerate in a neighbourhood of $\bar{X}$. Thus $D^{*} D$ is strongly elliptic there and we see that $\tilde{\imath}$ is of class $C^{1}$. This implies $\left.\imath^{\prime}\right|_{\partial X}=0$ as required.

To achieve the proof for a general case, we have only to know that the following Lemma holds.

Lemma 4. If $X$ is a strongly pseudoconvex manifold exhausted by $\dot{\varphi}$ as above, then there exist a closed analytic submanifold $\tilde{X}$ in a product $\boldsymbol{P}^{I I}(\boldsymbol{C}) \times \boldsymbol{C}^{N}$ of a projective space $\boldsymbol{P}^{M}(\boldsymbol{C})$ and $a \boldsymbol{C}^{N}$, and a proper holomophic map $\pi: \tilde{X} \rightarrow X$ which is biholomorphic outside a compact set. Furthermore $\tilde{X}$ can carry a Kähler metric with the condition (5.1). (Here $\lambda(\psi)$ is lifted to $\tilde{X}$ and $c_{2}$ is taken bigger if necessary.)

Proof. According to Grauert [8], there exists a compact analytic subset $A \subset X$ and a proper holomorphic map $\gamma$ from $X$ onto a Stein space $\hat{X}$ such that $\left.\gamma\right|_{x \backslash A}$ is biholomorphic. By the fundamental work of Hironaka [9], [10], [11], there is a complex manifold $\tilde{X}$ obtained from $\hat{X}$ by a succession of blowing-ups along nonsingular centers, such that the induced bimeromorphic map $\pi: \tilde{Y} \rightarrow X$ is holomorphic. $\tilde{X}$ can be chosen so that

(I) $\gamma \circ \pi$ is biholomorphic on $\tilde{X} \backslash \pi^{-1}(A)$,

(II) $\pi^{-1}(A)$ is a divisor with normal crossings whose irreducible components $\left\{\tilde{A}_{j}\right\}_{j=1}^{\nu}$ are smooth,

(III) there exist $\nu$ tuple of positive integers $\left(p_{1}, \cdots, p_{2}\right)$ so that the line bundle $\sum_{j=1}^{\nu} p_{j}[\tilde{A},]^{*}$ is very ample.

From this, the lemma follows immediately (for a similar argument, see the proof of Proposition 3.3 in [12] p. 231).

\section{§ 6. Conclusions}

We can now achieve the proof of our theorem. By [7], $\partial X$ can be realized as a hypersurface in a complex manifold $Y_{1}$ provided $\operatorname{dim}_{C} X \geqq 3$. Then a neighbourhood $W^{\gamma}$ of $\partial Y_{1}$ in $Y_{1}$ is separated into two parts by $\partial X$. Our Proposition 5 shows that one part has to be biholomorphic with $X$-(compact set). This means that we can patch $X$ and $W$ together and obtain a complex manifold $Y$, in which $X$ appears as a relatively compact open submanifold. By a suitable choice of $W$, we can identify the underlying differentiable manifold of $W$ with the previous M. ( $M$ may be replaced by a smaller one if necessary.) This means that the coordinate $u\left(=\frac{\xi 2 n}{5}\right)$ is a $C^{\infty}$-function $\tilde{\psi}$ on $Y$ which extends $\psi$ on $X$. Since the 
Levi form of $\psi$ remains positive definite at every point of $\partial X, \tilde{\varphi}$ is strictly plurisubharmonic on a neighbourhood of $\partial X$ in $Y$ and we have $X=\{x \in Y \mid \tilde{\psi}(x)<d\}$. This accomplishes the proof of our theorem.

As an application we shall give a consequence of our main theorem. Let $\mathscr{X}$ be the set of all complex structures on the underlying differentiable manifold $|X|$ of an spc domain $X$ in a complex manifold $M$. Let $\mathscr{X}_{0}$ be the subset of $\mathscr{X}$ which consists of the structures of finite distance from that of $X$ measured by the $C^{\infty}$-topology with respect to the metric on $M$. Then we have

Proposition 6. Let $X$ be an spc domain of dimension $\geqq 3$. Then there exists a neighbourhood $U$ of $X$ in $\mathfrak{X}_{0}$ such that every complex structure in $U$ provides on $|X|$ the structure of an spc domain.

The proof is immediate from our main theorem.

In conclusion, we express our thanks to Professor Norio Shimakura and other friends for their discussions. Thanks are also due to the referee for many suggestions.

\section{References}

[1] Aronszajn, N.A., A unique continuation theorem for solutions of elliptic partial differential equations or inequalities of the second order, J. Math. Pures Appl. 36 (1957), 235-249.

[2] Friedman, A., Partial differential equations, Holt. Rinehart and Winston, Inc., 1969.

[3] Hörmander, L., An introduction to complex analysis of several variables, Van Nostrand, 1966.

[4] Nakano, S., On the inverse of monoidal transformation, Publ. RIMS, Kyoto Univ., $6(1970-71), 483-502$.

[5] Ohsawa, T., Finiteness theorems on weakly 1-complete manifolds, Publ. RIMS, Kyoto Univ., 15 (1979), 853-870.

[6] - Cohomology vanishing theorems on weakly l-complete manifolds, Publ. RIMS, Kyoto Univ., 19 (1983), 1181-1201.

[7] - Global realization of strongly pseudoconvex $\mathrm{CR}$ manifolds, Publ. RIMS, Kyoto Univ, 20 (1984), 599-605.

[8] Grauert, H., On Levi's problem and the imbedding of real-analytic manifolds, Ann. of Math. 68 (1958), 460-472.

[9] Hironaka, H., Resolution of singularities of an algebraic variety over a field of characteristic zero I, II, Ann. of Math., 79 (1964), 109-326.

[10] Hironaka, H. and Rossi, H., On the equivalence of imbeddings of exceptional complex spaces, Math. Ann., 156 (1964), 313-333.

[11] Hironaka, H., Flattening theorems in complex-analytic geometry, Amer. J. Math. 97 (1975), 503-547.

[12] Wells, R.O., Differential analysis on complex manifolds, 2nd edition, GMT 65, Springer-Verlag New York Inc., 1980. 
